University of Warwick institutional repository: http://go.warwick.ac.uk/wrap This paper is made available online in accordance with publisher policies. Please scroll down to view the document itself. Please refer to the repository record for this item and our policy information available from the repository home page for further information.

To see the final version of this paper please visit the publisher's website. Access to the published version may require a subscription.

Author(s): A. CROS, R. ALI, P. LE GAL, P. J. THOMAS, L. SCHOUVEILER, P. W. CARPENTER and M. P. CHAUVE Article Title: Effects of wall compliance on the laminar-turbulent transition of torsional Couette flow

Year of publication: 2003

Link to published version: http://dx.doi.org/10.1017/S0022112003003999

Publisher statement: None 


\title{
Effects of wall compliance on the laminar-turbulent transition of torsional Couette flow
}

\author{
By A. CROS ${ }^{1}$, R. ALI I $I^{2}$ P. LE GAL ${ }^{1}$, P. J. THOMAS ${ }^{2}$, \\ L. SCHOUVEILER ${ }^{1}$, P. W. CARPENTER ${ }^{2}$ AND M. P. CHAUVE \\ ${ }^{1}$ Institut de Recherche sur les Phénomènes Hors Equilibre, UMR 6594, CNRS \& Universités \\ d'Aix-Marseille I et II, 49 rue F. Joliot Curie, BP 146, Technopôle de Château-Gombert, \\ 13384 Marseille Cédex 13, France \\ ${ }^{2}$ Fluid Dynamics Research Centre, School of Engineering, University of Warwick, \\ Coventry CV4 7AL, UK
}

(Received 24 June 2002 and in revised form 8 January 2003)

Torsional Couette flow between a rotating disk and a stationary wall is studied experimentally. The surface of the disk is either rigid or covered with a compliant coating. The influence of wall compliance on characteristic flow instabilities and on the laminar-turbulent flow transition is investigated. Data obtained from analysing flow visualizations are discussed. It is found that wall compliance favours two of the three characteristic wave patterns associated with the transition process and broadens the parameter regime in which these patterns are observed. The results for the effects of wall compliance on the third pattern are inconclusive. However, the experiments indicate that the third pattern is not a primary constituent of the laminar-turbulent transition process of torsional Couette flow.

\section{Introduction}

The concept of developing compliant coatings as a technique for laminar-flow control and, hence, for drag reduction has become widely known following the seminal publications of Kramer $(1957,1960,1962,1965)$. However, after the publication of his experimental results, dispute arose concerning the nature of compliant-wall/fluid-flow interactions and the feasibility of the suggested technique (Carpenter, Davies \& Lucey 2000).

Over the last twenty years it has been clearly established through experiments and theory that wall compliance can suppress the growth of Tollmien-Schlichting waves, leading to substantial delays in the onset of laminar-turbulent transition (see reviews by Riley, Gad-el-Hak \& Metcalfe 1988; Carpenter 1990; Carpenter et al. 2000; Carpenter, Lucey \& Davies 2001 and Gad-el-Hak 1996, 2003). However, this favourable evidence really only applies to low-noise flow environments, similar to the flat-plate boundary layer, where amplification of Tollmien-Schlichting waves is the primary route to transition. In many applications, other, quite different and much more powerful instabilities may be dominant and result in transition. An important example is the cross-flow vortices that develop near the leading edge of a swept wing. These also develop in the three-dimensional boundary layer over a rotating disk. This is one of the reasons why flow instability and transition in the rotating-disk flow has been widely investigated in the past. 
The influence of wall compliance on the transition of the rotating-disk boundary layer was studied theoretically by Cooper \& Carpenter $(1997 a, b)$ and Davies \& Carpenter (2001). A recent review of the subject is given by Carpenter, Thomas \& Nagata (2003). The first experiments exploring the effects of wall compliance on the instability modes were summarized by Colley et al. (1999). While they observed that wall compliance advanced transition to lower Reynolds numbers their data analysis revealed, nevertheless, a stabilizing influence on the cross-flow-vortex eigenmode (Type I instability in the rotating disk literature). The apparatus of Colley et al. was based on the facility of Jarre, Le Gal \& Chauve (1991, 1996a,b) and was intended to simulate the case of a disk spinning underneath a fluid that is at rest infinitely far above the disk surface. Colley et al. (2000) (summarized in Carpenter et al. 2003) carried out further experiments on the instability modes over compliant disks. They modified their previous apparatus to enable them to reduce the disturbance levels. The new results confirm that wall compliance stabilizes the Type I cross-flow vortices, but also show substantial delays in transition for the more compliant disks. Furthermore, the experimental data also reveal, in accordance with the theoretical predictions of Cooper \& Carpenter (1997a), that wall compliance destabilizes the viscous Type II eigenmode, so that it can be clearly detected at lower Reynolds numbers.

If the disk rotates inside an enclosure, in close proximity to a facing stationary wall parallel to the disk surface, then the flow is more complex. The disk then drives a recirculating flow inside the gap of width $h$ separating the disk from the wall. Boundary layers are established on both the disk and the stationary wall. If the gap width is sufficiently large in comparison to the combined thickness of these two boundary layers then both layers are separated from each other by a core of fluid that is almost in solid-body rotation (Batchelor 1951). The instabilities associated with this rotor-stator flow depend on the value of the ratio $\gamma=h / R$ of gap width and disk radius $R$ (Faller 1991). Different values of $\gamma$ result in different transition scenarios as was first noted by Daily \& Nece (1960). When the gap width is sufficiently small the boundary layers of rotor and stator merge. For very narrow gaps a quasilinear profile of the azimuthal component of the flow velocity develops and the flow configuration is referred to as torsional Couette flow. Torsional Couette flow was investigated experimentally by, for instance, San'kov \& Smirnov (1984), Chauve and Tavéra (1984), Itoh (1988) and Sirivat (1991).

Recently, transition of torsional Couette flow was studied in detail by Schouveiler, Le Gal \& Chauve (2001) and Cros \& Le Gal (2002) for configurations with gaps as narrow as $0.005 \leqslant \gamma \leqslant 0.02$. Their observations show that the onset of transition is characterized by a regular pattern of spiral vortices which appears once the rotation rate $F$ exceeds a certain critical threshold. Figure 1 $(a)$ illustrates the nature of the flow pattern. The spiral vortices seen in the figure are referred to as the SRIII (spiral) waves below. These waves for the torsional Couette flow were predicted computationally by Hoffman, Busse \& Chen (1998) who referred to them as 'Stationary Rolls'. They arise from viscous effects and from the Coriolis force and correspond to the wellknown Type II instability mode in the rotating-disk literature. Note that the SRIII waves also correspond to the waves labelled ' 1 ' in figure 1 of San'kov \& Smirnov (1991).

Other instabilities appear when the rotation rate $F$ is increased to higher values. The nature of the emerging instabilities depends on the parameter $\gamma$. When $\gamma$ is less than 0.016, turbulent spirals appear - figure 1(b). Cros \& Le Gal (2002) found that these turbulent spirals originate from defects appearing on the SRIII waves. The number of turbulent spirals decreases with the rotation rate $F$. These turbulent spirals may be 
(a)

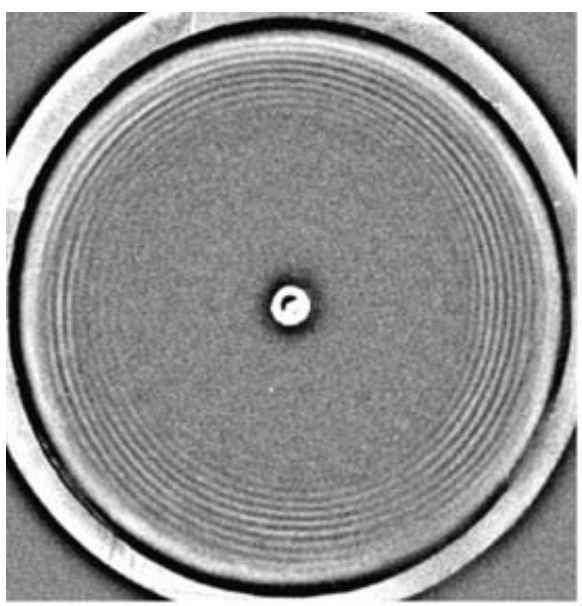

(c)

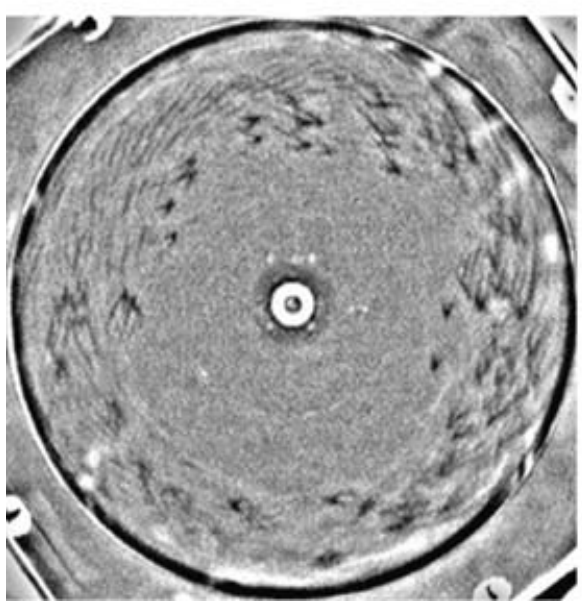

(b)

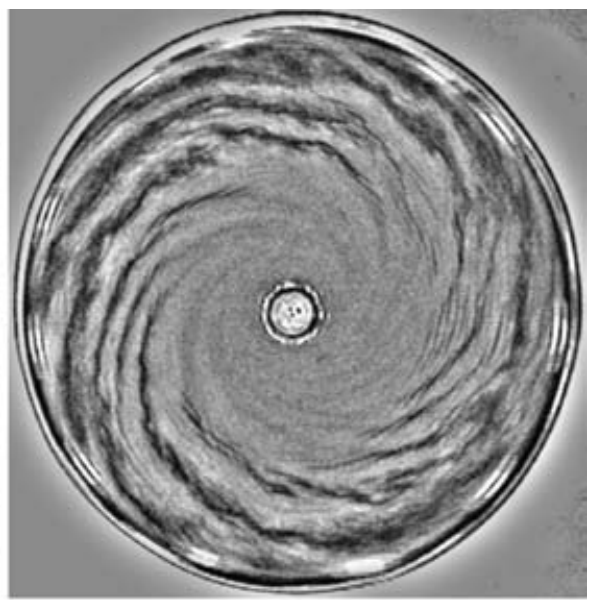

(d)

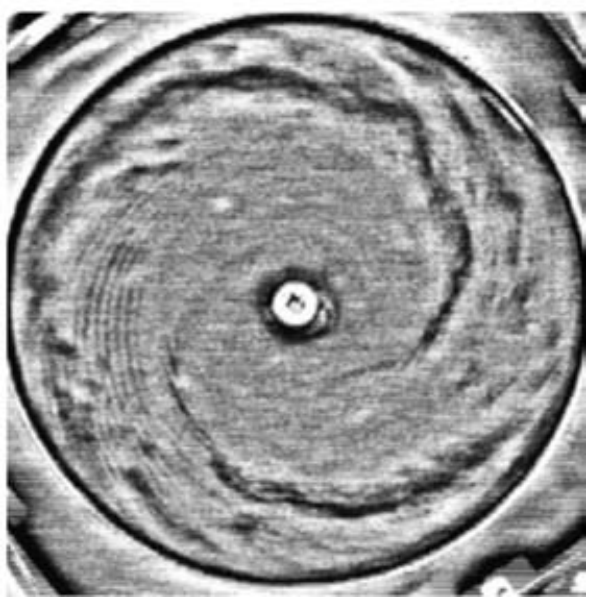

Figure 1. Torsional Couette flow visualizations from Cros \& Le Gal (2002) of $(a)$ the spiral waves SRIII ( $h=1.8 \mathrm{~mm}, F=38$ r.p.m. $),(b)$ turbulent spirals $(h=1.8 \mathrm{~mm}, F=74$ r.p.m. $)$, (c) spots $(h=2.5 \mathrm{~mm}, F=80$ r.p.m.), $(d)$ mixed states with spiral waves SRIII, spots and turbulent spirals $(h=2.1 \mathrm{~mm}, F=90$ r.p.m.). Clockwise rotation.

related to an unstable regime of the solitary vortices calculated by Hoffman \& Busse (2000). When $\gamma$ is above 0.016, spot-like structures appear - figure 1(c). These spots originate at the boundaries of the flow field and propagate along spiral trajectories towards the centre. The turbulent spots, as well as the solitary waves, represent isolated turbulent regions surrounded by laminar flow and, hence, characterize a spatiotemporal intermittent flow field. Note that the three structures (SRIII, turbulent spirals and spots) can co-exist in the flow as is shown on figure $1(d)$.

The analysis of their observations enabled Schouveiler et al. (2001) to construct transition diagrams for torsional Couette flow over rigid disks. The goal of the present study is to explore how wall compliance affects the emergence and disappearance of the observed characteristic flow structures and to construct a corresponding diagram for torsional Couette flow over a compliant disk. 


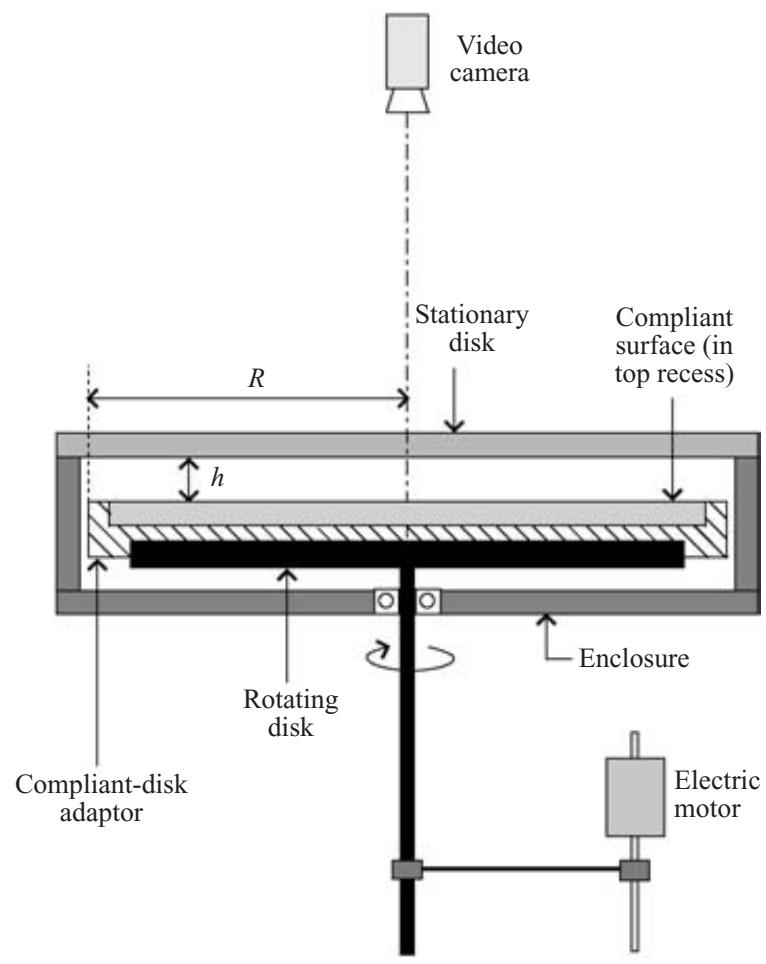

FIGURE 2. Sketch illustrating the modified experimental set-up with compliant-disk adapter mounted on the existing rotating disk, used in the study of Schouveiler et al. (2001).

\section{Experimental set-up and techniques}

Details of the experimental facility are described in Schouveiler et al. (2001). The apparatus was, however, slightly modified to enable testing of disks with compliant surfaces. Figure 2 schematically illustrates the modified arrangement.

Briefly, the facility consists of a belt-driven rotating disk spinning inside a waterfilled, stationary cylindrical enclosure. As it was not feasible to replace the original disk used by Schouveiler et al. (2001) by an alternative compliant disk, two disk adapters (diameter $2 R=298.9 \mathrm{~mm}$ ) were fabricated which could be mounted on top of the existing disk. One of the adapters was intended for experiments studying compliant disk surfaces. The other was to enable rigid-disk control experiments under otherwise identical (geometric) conditions. Figure 2 shows the compliant-disk adapter mounted on top of the facility's existing disk. This adapter is a circular aluminium disk with a top and a bottom recess. The bottom recess enables mounting the adapter on the existing disk inside the facility. Analogous to the disk used by Colley et al. (1999) the top recess $(8 \mathrm{~mm}$ deep) provides the space to accommodate a compliant coating. The only difference between this compliant-disk adapter and the rigid-disk adapter for the control experiments is that the latter did not have a top recess and, thus, provides a uniform, smooth $( \pm 5 \mu \mathrm{m})$ rigid surface.

The maximum rotation rate of the disk is $F=200$ r.p.m. and it can be kept constant to within an accuracy of $0.2 \%$. The rotational velocity $\Omega=2 \pi F$ is nondimensionalized and expressed in terms of a Reynolds number $R e=\Omega R^{2} / v$ with $v=1 \times 10^{-6} \mathrm{~m}^{2} \mathrm{~s}^{-1}$ the kinematic viscosity of water at the constant working-fluid temperature of $20^{\circ} \mathrm{C}$. 
The gap width can be adjusted continuously within $1.5 \mathrm{~mm} \leqslant h \leqslant 3 \mathrm{~mm}$. The spacing between the lateral cylindrical enclosure wall and the rotating disk is $2 \mathrm{~mm}$. The enclosure is manufactured from transparent acrylic sheet. Thus, the flow in the gap can be observed through the upper enclosure wall.

The water occupying the space above the rotating disk is seeded with Kalliroscope fluid to allow visualization of the structures developing in the flow field. Hence, the critical rotation rates $F$ for the emergence and disappearance of structures can be determined by a visual inspection of the flow. To this end, the flow was filmed with a CCD camera mounted vertically above the disk and co-rotating with it. Thus, the flow is observed in the rotating frame of reference. The error associated with the measurement of the rotation rate for the different thresholds by visual inspection of the flow is estimated to be \pm 3 r.p.m.

Compliant coatings were produced as described in detail by Colley et al. (1999). They were made from Silicon Gel (NuSil MED-6340, Polymer Systems Technology Ltd., High Wycombe, UK). After pouring the gel into the top recess of the compliantdisk adapter, it was covered with a smooth glass plate and placed inside an oven where it was allowed to cure for 5 days at an average temperature around $90-100^{\circ} \mathrm{C}$. The MED-6340 gel is supplied in two separate components. By varying their mixing ratio compliant coatings with different elastic properties can be produced. Two compliant disks were manufactured at the University of Warwick and then tested at IRPHE in Marseille.

The first disk (disk 1) was fabricated using a 1:1 mixing ratio of the two gel components. Its elastic properties were measured using the instrumentation and the techniques developed by Colley et al. (1999). The elastic modulus of this disk (disk 1) was $E=326 \mathrm{kPa}$ and its damping coefficient - using the nomenclature introduced by Colley et al. - was $\ln \left(h_{0} / h_{1}\right)=1.33 \pm 0.05$. These values are comparable to those of the disk used by Colley et al. (1999). The other disk (disk 2) had a higher level of compliance; it was produced from a $2: 1$ gel mixture $(2$ parts component $\mathrm{A}$ and 1 part component B). Its elastic modulus was $E=267 \mathrm{kPa}$ while its damping coefficient was $\ln \left(h_{0} / h_{1}\right)=3.11 \pm 0.05$.

We have estimated that the stresses arising from the centrifugal force at maximum rotation rates are sufficiently small to ensure that there is no appreciable deformation of the compliant coatings. While the gap width $h$ was manually adjusted to nominal values its exact value was measured by means of an optical technique involving the observation of multiple reflections of a laser beam originating from the successive solid-liquid interfaces within the facility.

\section{Experimental results}

\subsection{Rigid-disk control experiments}

As described in $\$ 2$, the facility of Schouveiler et al. (2001) was modified to enable experiments with compliant disks. Effects of wall compliance on the transition process are expected to be small. Hence, in order to exclude the possibility that measured differences between compliant-disk and rigid-disk control experiments merely reflect changes of the experimental conditions we initially determined a transition diagram for the rigid-disk flow in the modified facility. For each gap width $h$ data were obtained by increasing the rotation rate from rest to successively higher values.

The measured transition diagram is shown in figure 3. It is interpreted as follows. For instance, at $h=1.8 \mathrm{~mm}$ the flow is stable for rotation rates $F<45.2$ r.p.m. and, hence, no structures were visible in the flow field. Between 45.2 r.p.m. $\leqslant F<54.3$ r.p.m. 


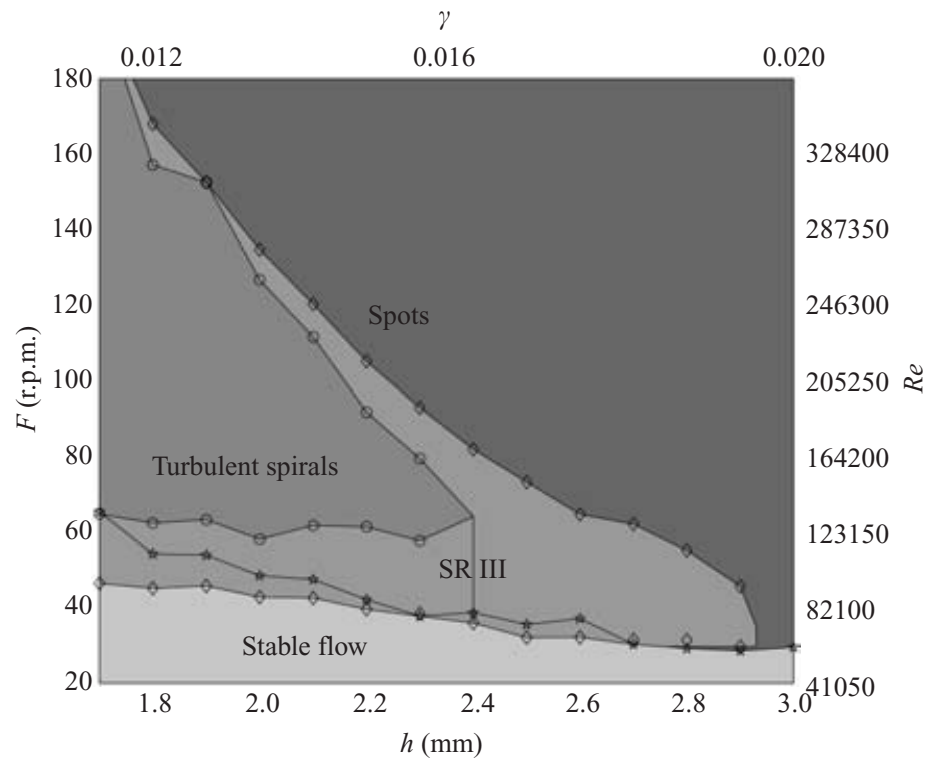

FIGURE 3. Transition diagram for torsional Couette flow over the rigid surface. $\diamond$, SRIII domain; $\bigcirc$, turbulent spiral domain; $\star$, threshold for the appearance of turbulent spots.

solely SRIII waves were observed. In the interval 54.3 r.p.m. $\leqslant F<62.5$ r.p.m. SRIII waves together with spots are visible. For 62.5 r.p.m. $\leqslant F<157.2$ r.p.m. SRIII waves, turbulent spirals and turbulent spots are present simultaneously. At $F \geqslant 168$ r.p.m. the SRIII waves and the turbulent spirals have disappeared and only turbulent spots remain visible in the flow field.

Similarly, at $h=2.6 \mathrm{~mm}$ the flow is stable for rotation rates $F<32.2$ r.p.m. Between 32.2 r.p.m. $\leqslant F<37.2$ r.p.m., only SRIII waves exist. For 37.2 r.p.m. $\leqslant F<64.8$ r.p.m. the SRIII waves coexist together with turbulent spots. Above $F=64.8$ r.p.m. only turbulent spots exist.

Overall, the new measurements reconfirm the data displayed in figure 3 of Schouveiler et al. (2001). A detailed comparison between the two diagrams reveals slight differences which are caused by the changes made to the experimental facility. Probably the most significant change is associated with the width of the clearance between the edge of the spinning disk and the vertical wall of the enclosure. In the modified set-up the clearance is $2 \mathrm{~mm}$ while it was only $0.05 \mathrm{~mm}$ in the original set-up.

\subsection{Compliant-disk experiments}

The rigid-disk data of $\S 3.1$ are compared with the data for the stiffer compliant disk 1 and the softer compliant disk 2. The data for SRIII spiral waves, turbulent spirals and turbulent spots are shown separately in figures $4(a), 4(b)$ and 5 respectively. Each plot displays the appropriate rigid-disk data of figure 3 together with the corresponding data sets for the two compliant disks.

Figure 4(a) shows that, within the measurement accuracy of \pm 3 r.p.m., the data for SRIII waves for rigid-disk flow and for the flow over the stiff compliant disk 1 essentially coincide. The data points for the compliant disk 1 on the upper boundary identifying the disappearance of the spiral waves - are consistently located below the data for the rigid disk. However, the differences are not substantial enough to associate them with effects of wall compliance. 

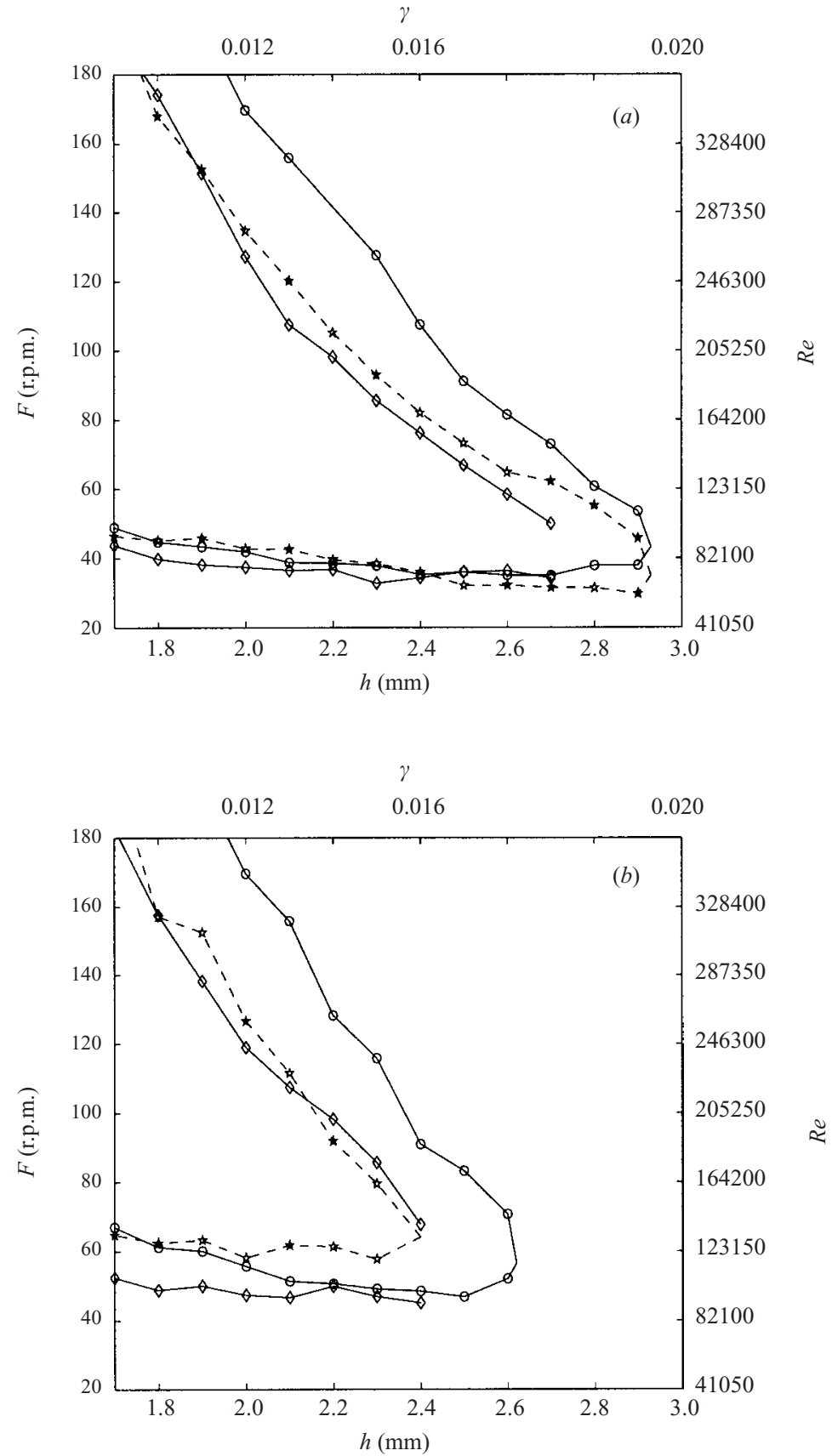

FIGURE 4. Diagram illustrating the influence of wall compliance on the emergence and disappearance of $(a)$ the SRIII spiral waves and $(b)$ turbulent spirals. $\star$, rigid disk; $\diamond$, compliant disk 1; O, compliant disk 2.

Significant differences are revealed by comparing the rigid-disk data and the results for compliant disk 1 with the data for the softer compliant disk 2. The onset for the emergence of the SRIII waves remains unaffected when the compliance level is increased. However, the waves now disappear at critical rotation rates that are as

\section{CAMBridge JDUNALS}




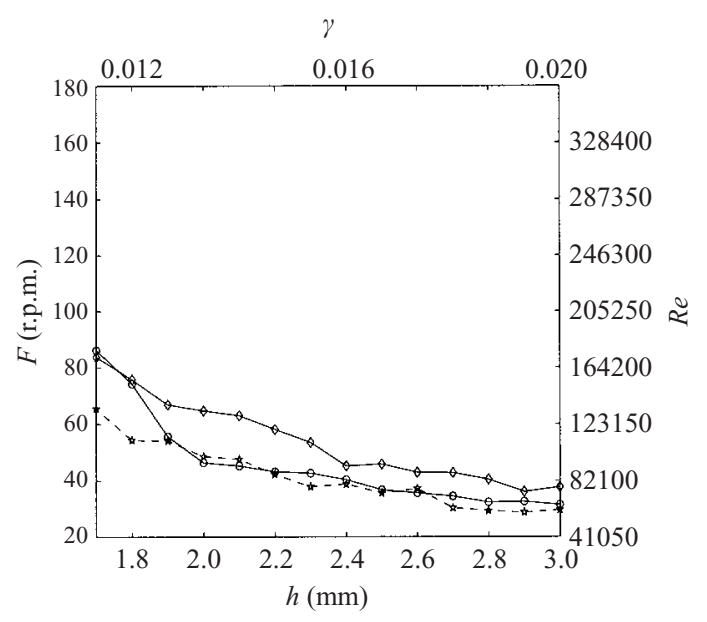

FIGURE 5. Diagram illustrating the influence of wall compliance on the emergence of the turbulent spots. $\star$, rigid disk; $\diamond$, compliant disk 1 ; $\bigcirc$, compliant disk 2 .

much as 30 r.p.m. higher than corresponding values for flow over the rigid disk and over compliant disk 1 . Hence, wall compliance favours the occurrence of SRIII waves and broadens the parameter regime in which they are observed.

The turbulent spirals shown in figure $1(b)$ evolve from defects in the SRIII spiral wave pattern. Figure $4(b)$ compares the critical rotational rates for the appearance and disappearance of the turbulent spirals. The figure shows that wall compliance advances the appearance of the turbulent spirals in comparison to the rigid-disk flow. Also, the disappearance of the turbulent spirals is delayed substantially. The delay is as large as 30 r.p.m. for compliant disk 2 in comparison to the corresponding values for flow over the rigid disk and compliant disk 1 . Thus, corresponding to the results revealed by the transition diagram for SRIII waves in figure 4(a) the overall effect of wall compliance is to broaden the stability regime of the turbulent spirals.

Figure 5 displays the critical rotation rates for the emergence of the turbulent spots over the rigid disk and over the two compliant disks. The data for the softer disk 2 essentially coincide with the rigid disk thresholds, while thresholds for the stiffer disk 1 are up to approximately 30 r.p.m. higher. Thus, the results revealed by the data in figure 5 seem inconclusive.

It is possible for low values of compliance to have a greater effect on certain classes of disturbance than higher values. This is seen, for example, in the theoretical results of Cooper \& Carpenter (1997a), showing low levels of compliance greatly advancing the onset of the viscous Type II eigenmodes, whereas for higher levels the results were little different from those for a rigid disk. This occurs when there are two competing mechanisms, one of which is suppressed by wall compliance, while the other is strengthened. However, we believe that the differences between the data for the rigid disk and compliant disk 1 are more likely to be due to other factors than wall compliance. Our observations have shown that the spots originate from locations at the edge of the disk. They are generated in the immediate vicinity of the thin, vertical gap separating the rotating disk from the vertical wall of the stationary enclosure. The critical rotation rate for the emergence of the spots was found to be very sensitive to the boundary conditions encountered in this region. This suggests that the spots are introduced into the flow as a result of disturbances associated with the finite size of the experimental facility. Hence, we believe that the spots are not 
primary constituents of the laminar-turbulent transition process of torsional Couette flow.

\section{Summary and conclusion}

In order to understand and interpret our experimental results, it should be noted that the effective compliance increases with linear speed. Thus in the present context the wall compliance is best characterized by introducing a coefficient corresponding to the non-dimensional elastic modulus, for example, either

$$
C_{E} \equiv \frac{E}{\rho \Omega^{2} R^{2}} \quad \text { or } \quad C_{E} \equiv \frac{E}{\mu \Omega},
$$

where $\rho$ and $\mu$ are respectively the density and dynamic viscosity of the water. The first definition would be suitable for boundary-layer flows, the second is probably more appropriate for the viscous-dominated torsional Couette flow. In both cases the effective compliance rises $\left(C_{E}\right.$ falls) as the rotational speed increases.

The SRIII waves and the turbulent spirals shown in figures $1(a)$ and $1(b)$ are visualizations of characteristic intermediate flow stages associated with the laminarturbulent transition process of torsional Couette flow. Our experimental results presented in figures $4(a)$ and $4(b)$ can be interpreted as showing that wall compliance has little effect on either of these classes of disturbances until a certain threshold value is reached. Thus in figure $4(a)$ the lower boundary corresponding to the onset of the disturbances appears to be largely unaffected by compliance. But this boundary is found only at low values of rotational speed where the effective compliance is low. It is only the upper boundary corresponding to the more compliant disk at much higher rotational speeds that is clearly shifted upward compared with the rigid-wall results. The results in figure $4(b)$ are similar, except that the lower boundary appears to be more affected by compliance.

Overall our results suggest that wall compliance enhances the mechanisms responsible for sustaining both the SRIII waves and the turbulent spirals, thereby broadening the parameter regimes over which they are observed. However, the advanced stages of the transition process of the flow are characterized by the appearance of the turbulent spots shown in figure 1(c). These spots originate from locations near the enclosure wall, at the boundary of the flow field, from where they propagate radially inwards. Our observations suggest that these spots are not primary constituents of the laminar-turbulent transition process of torsional Couette flow. Most likely they represent disturbances introduced into the flow as a result of the finite size of the experimental facility. Since the spots are very sensitive to the boundary conditions near the enclosing walls of the facility our experiments were inconclusive regarding their interaction with wall compliance.

This research is funded by the Royal Society (UK) and the CNRS (France) through a Joint Project Grant.

\section{REFERENCES}

BATCHELOR, G. K. 1951 Note on a class of solutions of the Navier-Stokes equations representing steady rotationally-symmetric flow. Q. J. Mech. Appl. Maths 4, 29-41.

Carpenter, P. W. 1990 Status of transition delay using compliant walls. Prog. Astro. Aero. 79, $758-765$.

Carpenter, P. W., Davies, C. \& Lucey, A. D. 2000 Hydrodynamics and compliant walls: Does the dolphin have a secret? Curr. Sci. 79, 758-765. 
Carpenter, P. W., Lucey, A. D. \& Davies, C. 2001 Progress on the use of compliant walls for laminar-flow control. J. Aircraft 38, 504-512.

Carpenter, P. W., Thomas, P. J. \& Nagata, M. 2003 Rotating flows over compliant walls. In Flow Past Highly Compliant Boundaries and in Collapsible Tubes (ed. P. W. Carpenter \& T. J. Pedley), pp. 167-188. Kluwer.

Chauve, M. P. \& Tavéra, G. 1984 Rotating disk flows, transition to turbulence. In Cellular Structures in Instabilities (ed. J. E. Wesfreid \& S. Zaleski), Lecture Notes in Physics, vol. 210, pp. 307-318. Springer.

Colley, A. J., Carpenter, P. W. \& Thomas, P. J. 2000 Experiments on boundary-layer transition over a rotating disc - New experimenatal method. 4th EUROMECH Fluid Mechanics Conference, Eindhoven, The Netherlands, 19-23 November.

Colley, A. J., Thomas, P. J., Carpenter, P. W. \& Cooper, A. J. 1999 An experimental study of boundary-layer transition over a rotating disk. Phys. Fluids 11, 3340-3352.

COOPER, A. J. \& CARPENTER, P. W. 1997a The stability of the rotating-disk boundary-layer flow over a compliant wall. Part 1. Type I and Type II instabilities, J. Fluid Mech. 350, 231-259.

CoOper, A. J. \& CARPenter, P. W. $1997 b$ The stability of the rotating-disk boundary-layer flow over a compliant wall. Part 2. Absolute instability, J. Fluid Mech. 350, 261-270.

Cros, A. \& Le GaL, P. 2002 Spatio-temporal intermittency in the torsional Couette flow between a rotating and a stationary disk. Phys. Fluids 14, 3755-3765.

Daily, J. W. \& NeCE, R. E. 1960. Chamber dimension effects on induced flow and frictional resistance of enclosed rotating disks. Trans. ASME: J. Basic Engng 82, 217-232.

DAvies, C. \& CARPENTER, P. W. 2001 A novel velocity-vorticity formulation of the Navier-Stokes equations with applications to boundary-layer disturbance evolution, J. Comput. Phys. 172, $119-165$.

FALler, A. J. 1991 Instability and transition of disturbed flow over a rotating disk. J. Fluid Mech. 230, 245-269.

Gad-El-HaK, M. 1996 Compliant coatings: A decade of progress. Appl. Mech. Rev. 49, S1-S11.

GaD-EL-HaK, M. 2003 Drag reduction using compliant walls. In Flow Past Highly Compliant Boundaries and in Collapsible Tubes (ed. P. W. Carpenter \& T. J. Pedley). Kluwer.

Hoffmann, N. \& Busse, F. H. 2000 Isolated solitary vortex solutions for the Ekman-Couette layer. Eur. J. Mech. B/Fluids 19, 391-402.

Hoffmann, N., Busse, F. H. \& Chen, W.-L. 1998 Transition to complex flows in the Ekman-Couette layer. J. Fluid Mech. 366, 311-331.

Iтон, M. 1988 Instability and transition of the flow around a rotating disk in a casing. Toyota Rep. 36, 28-36.

Jarre, S., Le Gal, P. \& Chauve, M. P. 1991 Experimental analysis of the instability of the boundary layer over a rotating disk. Europhys. Lett. 14, 649-654.

Jarre, S., Le Gal, P. \& Chauve, M. P. $1996 a$ Experimental study of rotating disk instability. I. Natural flow. Phys. Fluids 8, 496-508.

Jarre, S., Le Gal, P. \& Chauve, M. P. $1996 b$ Experimental study of rotating disk flow instability. II. Forced flow. Phys. Fluids 8, 2985-2994.

Kramer, M. O. 1957 Boundary-layer stabilization by distributed damping. J. Aero. Sci 24, 459-460.

Kramer, M. O. 1960 Boundary layer stabilization by distributed damping. Nav. Engng J. 72, 25-33.

Kramer, M. O. 1962 Boundary layer stabilization by distributed damping. Nav. Engng J. 74, $341-348$.

Kramer, M. O. 1965 Hydrodynamics of the dolphin. Adv. Hydrosci. 2, 111-130.

Riley, J. J., Gad-El-HaK, M. \& Metcalfe, M. 1988 Compliant coatings. Annu. Rev. Fluid Mech. 20, 393-420.

SAN'Kov, P. I. \& Smirnov, E. M. 1984 Bifurcation and transition to turbulence in the gap between rotating and stationary parallel disks. Fluid. Dyn. 19, 695-702.

SAN'Kov, P. I. \& SMIRnov, E. M. 1991 Stability of viscous flow between rotating and stationary disks. Fluid. Dyn. 26, 857-864.

Schouveiler, L., Le Gal, P. \& Chauve, M. P. 2001 Instabilities of the flow between a rotating and a stationary disk. J. Fluid Mech. 443, 329-350.

Sirivat, A. 1991 Stability experiment of flow between a stationary and a rotating disk. Phys. Fluids A 3, 2664-2671. 